04

\title{
Экспериментальное исследование изображающего объектива на основе дифракционных линз, корректирующих аберрации
}

\author{
(C) Р.В. Скиданов ${ }^{1,2}$, С.В. Ганчевская ${ }^{1,2}$, В.С. Васильев ${ }^{1}$, В.В. Подлипнов ${ }^{1,2}$ \\ ${ }^{1}$ ИСОИ РАН - филиал ФНИЦ „Кристаллография и фотоника“ РАН, \\ 443001 Самара, Россия \\ ${ }^{2}$ Самарский национальный исследовательский университет имени академика С.П. Королёва, \\ 443086 Самара, Россия \\ e-mail: romans@ipsiras.ru
}

Поступила в редакцию 16.12.2020 г.

В окончательной редакции 16.12.2020 г.

Принята к публикации 22.12.2020 г.

Рассмотрен простейший объектив, состоящий из рефракционной и дифракционной линз, в котором за счет формы дифракционной линзы компенсируются основные геометрические аберрации. Рассмотрен способ расчета такой системы на основе минимизации хроматической аберрации. Представлены результаты эксперимента, в котором напрямую определялась частотно-контрастная характеристика гибридного объектива.

Ключевые слова: дифракционная линза, гибридный объектив, хроматическая аберрация, геометрические аберрации.

DOI: $10.21883 /$ OS.2021.04.50772.304-20

\section{Введение}

В современных смартфонах используется все более сложная оптика для получения высокого качества изображения. Поскольку толщина корпуса уже не позволяет уместить такие объективы при нормальном расположении, в последних моделях смартфонов используется конфигурация с поворотной призмой. Минимизация размеров объектива является актуальной задачей, без решения которой невозможно дальнейшее сокращение размеров таких устройств. Минимизировать размеры объектива можно, использовав дифракционную или гармоническую линзу [1-8]. За счет особой формы таких линз можно уменьшить хроматизм, но все же относительно высокая хроматическая аберрация этих оптических элементов должна устраняться дополнительной цифровой обработкой [9]. С одной стороны, дополнительная обработка вполне подходит для регистрирующих устройств в современных смартфонах, обладающих высокими вычислительными мощностями, с другой стороны, оптическое разрешение в современных смартфонах настолько высоко, что цифровой обработки недостаточно, и желательно использование объектива, сопоставимого по качеству изображения с объективами классического типа на основе большого количества рефракционных линз. Все чаще рассматриваются гибридные системы, содержащие как рефракционные, так и дифракционные элементы [10-15]. Однако работы по гибридным системам не используют все возможности коррекции аберраций на основе дифракционных линз. Дифракционные линзы в таких системах используются классическим образом как некий ахроматизирующий элемент в составе классического многолинзового объ- ектива [10] либо в сочетании с рефракционным элементом свободной формы [12], который компенсирует геометрические аберрации в объективе. Также используются принципиально двухфокусные системы $[14,15]$. Ни в одной работе не попытались модифицировать рельеф дифракционной линзы для компенсации основных геометрических аберраций. В настоящей работе сделана попытка объединить компенсацию хроматических и геометрических аберраций в одной дифракционной линзе.

\section{1. Основные формулы}

Основная идея использования дифракционной оптики в составе классических изображающих объективов основана на том, что материальная дисперсия рефракционных линз и дисперсия фокусирующих свойств дифракционной линзы имеют разные знаки. С увеличением длины волны фокусное расстояние рефракционной линзы увеличивается, для дифракционной же линзы наблюдается обратный эффект. Использование дублета (рис. 1) из рефракционной и дифракционной линз позволяет при правильном подборе параметров полностью исключить хроматическую аберрацию на двух длинах волн и уменьшить ее в интервале между этими длинами волн.

Данная схема рассматривается исключительно как в принципе не пригодная к практическому использованию модель, поскольку при использовании стандартной сферической рефракционной линзы и стандартной дифракционной линзы, аппроксимирующей подобную же сферическую линзу, получается система с существенными геометрическими аберрациями. Поэтому дифракционная 


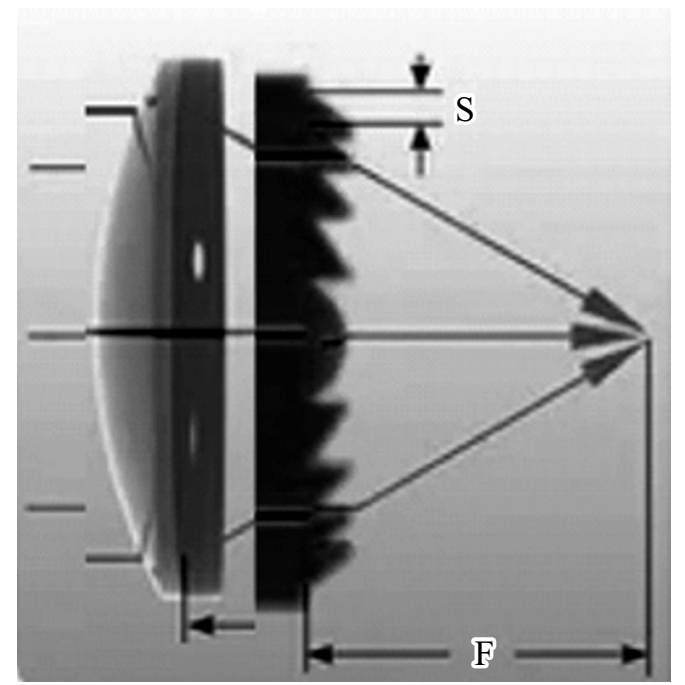

Рис. 1. Классическая иллюстрация дублета из рефракционной и дифракционной линз, в котором компенсируется хроматическая аберрация.

оптика рассматривается в составе достаточно сложных оптических систем [7,8] лишь как элемент компенсации хроматической аберрации. Однако возможности дифракционной оптики не ограничиваются только компенсацией хроматических аберраций. Особое внимание следует обратить на результаты работы [9], где для компенсации геометрических аберраций использовали сложнейший рефракционный элемент свободной формы, a в оптической системе находится и дифракционная линза. Эта работа хорошо иллюстрирует подход исследователей, хорошо знакомых с расчетом рефракционных систем и не использующих все возможности дифракционной оптики. Дифракционная линза может аппроксимировать расположением зон любую асферическую поверхность сколь угодно высокой сложности, что позволяет использовать ее одновременно и как компенсатор геометрических аберраций, и дифракционный хроматизм от этой формы практически не зависит.

Рассмотрим основные расчетные формулы для формирования рефракционно-диффракционного ахроматического дублета. Условие ахроматизации - это равенство фокусных расстояний системы из двух линз при двух длинах волн. Для рефракционной линзы фокусное расстояние на длине волны $\lambda_{1}$ будет определяться формулой

$$
f\left(\lambda_{1}\right)=\frac{R_{1} R_{2} n\left(\lambda_{1}\right)}{\left[n\left(\lambda_{1}\right)-1\right]\left[n\left(\lambda_{1}\right)\left(R_{1}+R_{2}\right)-d\left(n\left(\lambda_{1}\right)-1\right)\right]},
$$

где $R_{1}$ - радиус кривизны первой поверхности, $R_{2}-$ радиус кривизны второй поверхности, $d-$ толщина линзы, $n\left(\lambda_{1}\right)$ - показатель преломления на длине волны $\lambda_{1}$.
Для дифракционной линзы фокусное расстояние будет определяться как

$$
f^{d}\left(\lambda_{1}\right)=\frac{\lambda_{0} f_{0}}{\lambda_{1}}
$$

где $\lambda_{0}-$ расчетная длина волны, $f_{0}-$ расчетное фокусное расстояние. Аналогично для длины волны $\lambda_{2}$

$$
\begin{gathered}
f\left(\lambda_{2}\right)=\frac{R_{1} R_{2} n\left(\lambda_{2}\right)}{\left[n\left(\lambda_{2}\right)-1\right]\left[n\left(\lambda_{2}\right)\left(R_{1}+R_{2}\right)-d\left(n\left(\lambda_{2}\right)-1\right)\right]}, \\
f^{d}\left(\lambda_{2}\right)=\frac{\lambda_{0} f_{0}}{\lambda_{2}} .
\end{gathered}
$$

Совокупное фокусное расстояние системы из дифракционной и рефракционной линз выражается формулой

$$
F=\frac{f f^{b}}{f+f^{d}-D} .
$$

Условие равенства общего фокусного расстояния

$$
\frac{f\left(\lambda_{1}\right) \lambda_{0} f_{0}}{\lambda_{1}\left[f\left(\lambda_{1}\right)+\frac{\lambda_{0} f_{0}}{\lambda_{1}}-D\right]}=\frac{f\left(\lambda_{2}\right) \lambda_{0} f_{0}}{\lambda_{2}\left[f\left(\lambda_{2}\right)+\frac{\lambda_{0} f_{0}}{\lambda_{2}}-D\right]} .
$$

После несложных преобразований получаем

$\lambda_{0} f_{0}=\frac{f\left(\lambda_{1}\right) f\left(\lambda_{2}\right)\left(\lambda_{1}-\lambda_{2}\right)-d\left[f\left(\lambda_{2}\right) \lambda_{2}-f\left(\lambda_{1}\right) \lambda_{1}\right]}{f\left(\lambda_{1}\right)-f\left(\lambda_{2}\right)}$.

Очевидно, что наилучший результат достигается, если расчетная длина волны будет располагаться между $\lambda_{1}$ и $\lambda_{2}, \lambda_{0}=\frac{\lambda_{1}+\lambda_{2}}{2}$, тогда формула (7) преобразуется к виду

$$
f_{0}=\frac{2\left\{f\left(\lambda_{1}\right) f\left(\lambda_{2}\right)\left(\lambda_{1}-\lambda_{2}\right)-d\left[f\left(\lambda_{2}\right) \lambda_{2}-f\left(\lambda_{1}\right) \lambda_{1}\right]\right\}}{\left(\lambda_{1}+\lambda_{2}\right)\left[f\left(\lambda_{1}\right)-f\left(\lambda_{2}\right)\right]} .
$$

Расчет фазовой функции дифракционной линзы проводился исходя из простого условия: неидеальный волновой фронт $\varphi_{R}(r)$ от падающего на систему пучка, параллельного оптической оси, сформированный рефракционной линзой, после прохождения через дифракционную линзу должен стать идеальным сферическим волновым фронтом $\varphi_{0}(r)$, сходящимся точно в точку пересечения фокальной плоскости системы с оптической осью. Таким образом, фазовая функция линзы будет определяться разностью фазовых функций сформированного рефракционной линзой и идеального волновых фронтов

$$
\varphi^{d}(r)=\varphi_{0}(r)-\varphi_{R}(r),
$$

где $r$ - расстояние от оптической оси.

\section{2. Моделирование}

Первоначально была рассчитана система с оптическими параметрами, соответствующими среднему объективу смартфона. На рис. 2 представлена одна из возможных конфигураций объектива $f=3.5 \mathrm{~mm}$ (эквивалент 


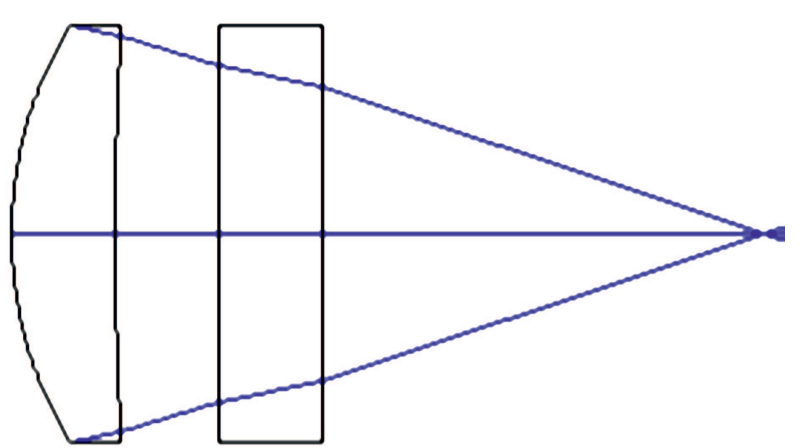

Рис. 2. Гибридная система из рефракционной и дифракционной линз с фокусным расстоянием $3.5 \mathrm{~mm}$.

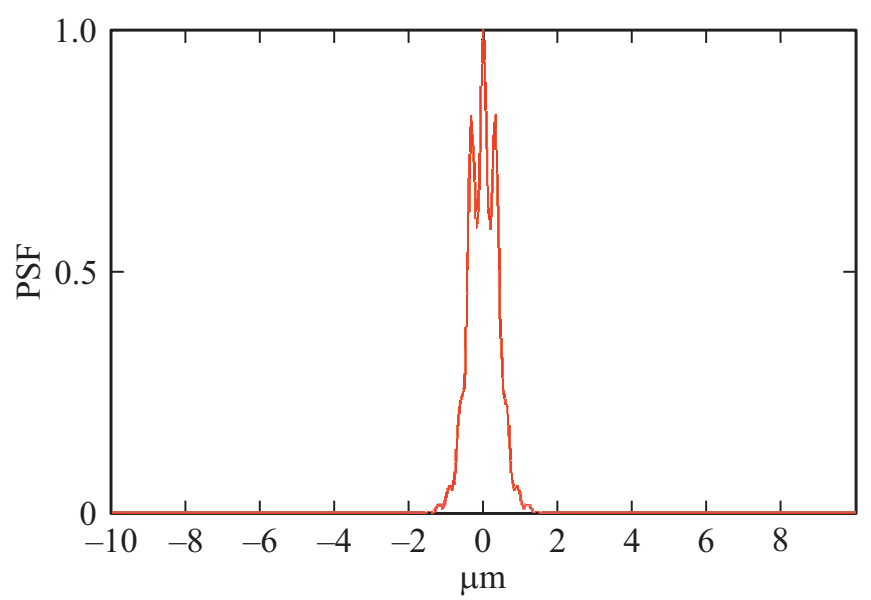

Рис. 3. Функция рассеяния точки гибридного объектива с фокусным расстоянием $3.5 \mathrm{~mm}$ : зависимость интенсивности света от пространственной координаты в направлении, перпендикулярном оптической оси.

28 mm при кроп-факторе 8), выполненная в коммерческом пакете ZEMAX. Устанавливается первая линза обычная сферическая, с $R_{1}=1.9 \mathrm{~mm}, R_{2}=16.7 \mathrm{~mm}$, толщиной $0.5 \mathrm{~mm}$ (стекло ВK7), на расстоянии $0.5 \mathrm{~mm}$ от нее располагается дифракционная линза на подложке из того стекла и той же толщины. Микрорельеф линзы на пленке ПММА толщиной $0.81 \mu \mathrm{m}$. Дифракционная линза является аппроксимацией линзы, полученной по формуле (9).

К сожалению, ZEMAX не позволяет работать с нестандартными дифракционными линзами (на рис. 2 использована стандартная линза Френеля), поэтому для расчета функции рассеяния точки (ФРТ) сформированной гибридной системы было разработано специальное программное обеспечение, которое позволило определить ФРТ. На рис. 3 представлена рассчитанная в этой программе ФРТ для объектива по диапазону длин волн от 400 до $700 \mathrm{~nm}$.

Как видно из рис.3, ширина ФРТ получившейся гибридной системы составляет $0.9 \mu \mathrm{m}$, что примерно соответствует дифракционному пределу.

\section{3. Изготовление дифракционной линзы}

Для проведения эксперимента промоделированная гибридная система не подходит из-за технических проблем c реализацией экспериментальной оптической схемы. Имеющиеся у экспериментаторов светочувствительные матрицы существенно больше, чем матрицы, используемые в смартфонах. Для расчета экспериментальной системы в качестве основы была выбрана рефракционная линза с точно известными параметрами: $f=30 \mathrm{~mm}$, двояковыпуклая с одинаковыми радиусами кривизны из стекла BK7. Расчет велся для диапазона длин волн от 450 до $1000 \mathrm{~nm}$. В качестве расчетной длины волны была использована длина волны в середине этого диапазона $-725 \mathrm{~nm}$. Поскольку линза формировалась на фоторезисте с показателем преломления 1.64, расчетная высота микрорельефа составила $1130 \mathrm{~nm}$. Это позволило точно рассчитать параметры волнового фронта, формируемого линзой на расстоянии $5 \mathrm{~mm}$ от ее задней главной плоскости, и на основе расчетов сформировать фазовую функцию дифракционной линзы. Из-за причин технологического характера фазовую добавку для компенсации волнового фронта (9) решено было реализовать в виде огибающей дифракционного микрорельефа (рис. 4). Методом прямой лазерной записи по фоторезисту [16] была изготовлена дифракционная линза, профиль которой представлен на рис. 4. Фокусное расстояние линзы составило около $1200 \mathrm{~mm}$. Высота микрорельефа линзы ближе к центру, к сожалению, немного выше расчетной (около $1400 \mathrm{~nm}$ ), но начиная с радиуса $1500 \mu \mathrm{m}$ совпадает с расчетным значением с ошибкой не более $10 \%$. Учитывая, что через область радиусом $1500 \mu \mathrm{m}$ проходит менее $10 \%$ световой энергии, эта ошибка не должна оказать заметного влияния на качество формируемого изображения.

Совокупное фокусное расстояние образованной рефракционной и дифракционной линзами системы составило $29.25 \mathrm{~mm}$, что не сильно отличается от исходного фокусного расстояния и позволяет в дальнейшем сравнивать результаты работы одиночной рефракционной линзы и разработанной гибридной системы.

\section{4. Эксперимент}

Для проведения эксперимента была собрана оптическая схема, представленная на рис. 5.

Проверка работы гибридного объектива предполагалась в широкоугольном режиме, поэтому мира шириной $300 \mathrm{~mm}$ располагалась на расстоянии всего $800 \mathrm{~mm}$ от объектива (поле зрения около $20^{\circ}$ ). На рис. 6 представлено изображение одной из мир, использованных в экспериментах. Использовано три полосатых миры с разными положениями относительно оптической оси системы. 


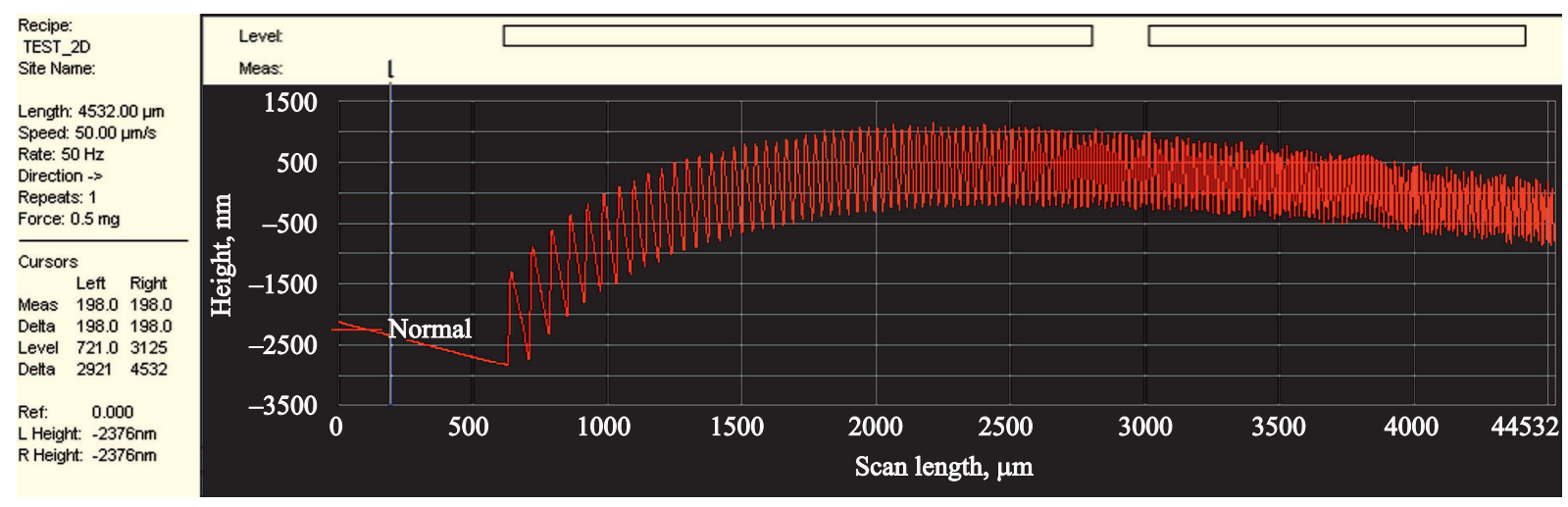

Рис. 4. Профилограмма изготовленной дифракционной линзы с фокусным расстоянием $1190 \mathrm{~mm}$.

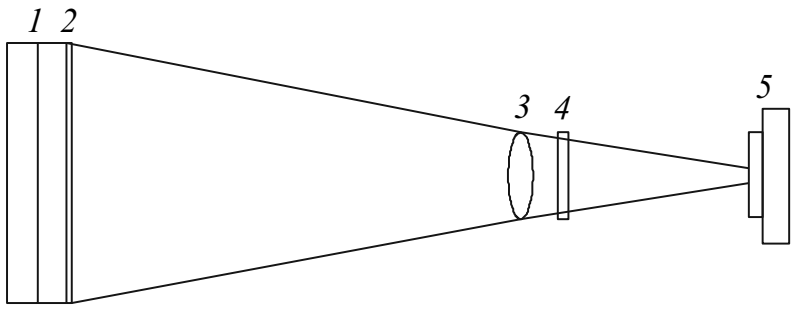

Рис. 5. Схема оптической установки: 1 - светодиодная панель с равномерной яркостью, 2 - пленка со светотехнической мирой, 3 - рефракционная линза $f=30 \mathrm{~mm}, 4$ - дифракционная линза $f=1190 \mathrm{~mm}, 5-$ светочувствительная матрица.

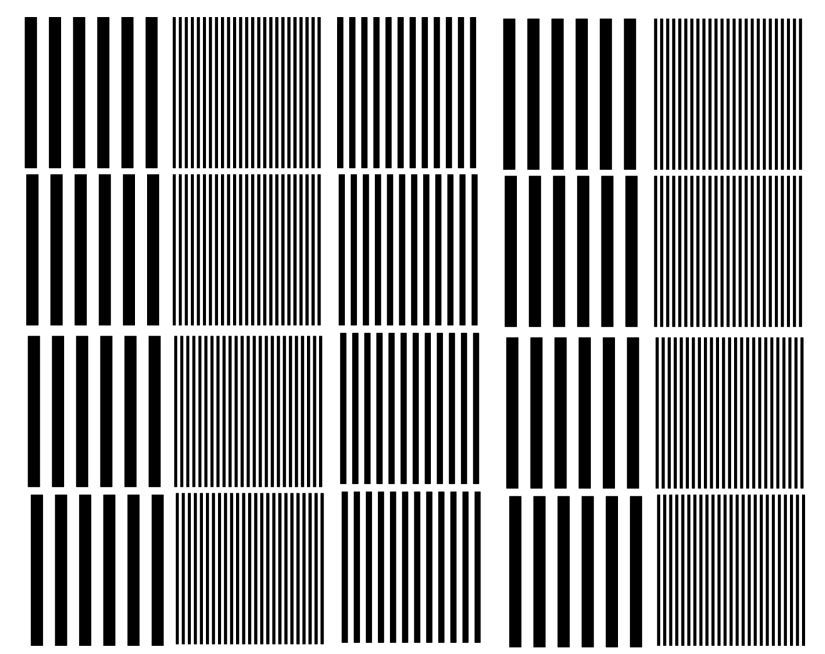

Рис. 6. Мира для эксперимента с гибридным объективом.

Для эксперимента разработано специальное программное обеспечение, которое на основе полученных изображений мир рассчитывало контраст изображения полос разной частоты.

На рис. 7 представлено изображение участка миры и его сечение в программном обеспечении, по которому и вычислялся контраст в зависимости от частоты линий (частотно-контрастная характеристика, ЧКХ).
Из рис. 7 видно, что гибридная система наиболее хорошо работает в области вблизи оптической оси (более низкочастотные линии слева имеют меньший контраст, чем более высокочастотные в центре).

На основе серии экспериментов с разными мирами были получены ЧКХ для гибридной системы и для одиночной рефракционной линзы. На рис. 8 приведены экспериментальные ЧКХ для гибридной системы, частоты даны на растр светочувствительной матрицы (ширина $10 \mathrm{~mm}$, размер пикселя $4.75 \mu \mathrm{m})$.

Среднее значение контраста по серии экспериментов составило 0.17. Для одиночной рефракционной линзы среднее значение контраста 0.14. При сужении поля зрения с $20^{\circ}$ до $10^{\circ}$ средние значения контраста составили 0.22 и 0.16 соответственно. Таким образом, при сужении поля зрения преимущество такой гибридной системы перед одиночной рефракционной линзой возрастает. Набор линий на рис. 8 на частоте около 90 образовался из-за
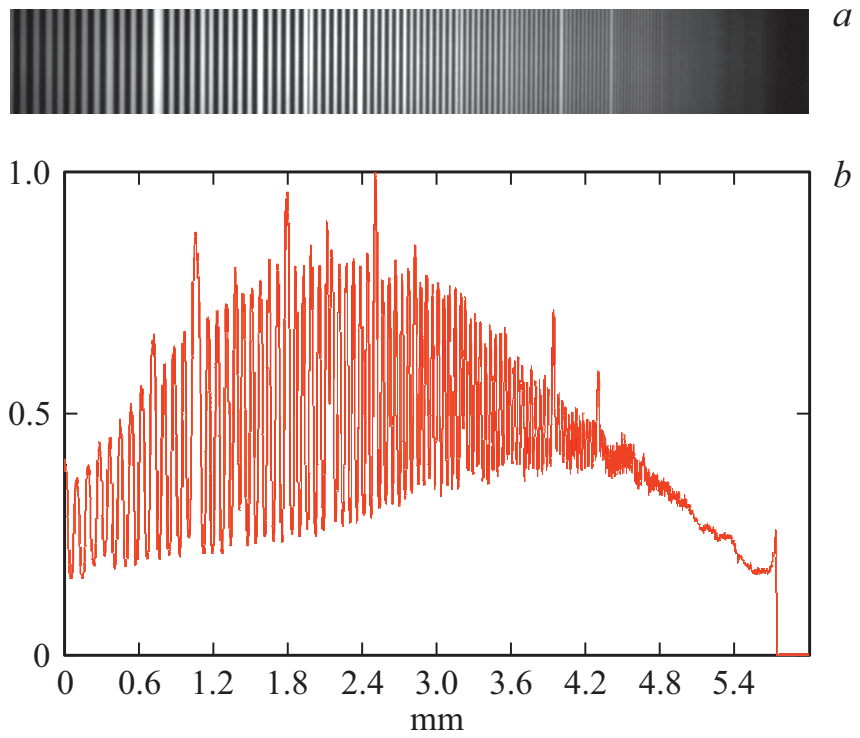

Рис. 7. Изображение участка миры $(a)$ и сечение (зависимость интенсивности от пространственной координаты от 0 до $0.95 \mathrm{~mm})$ этого изображения $(b)$. 


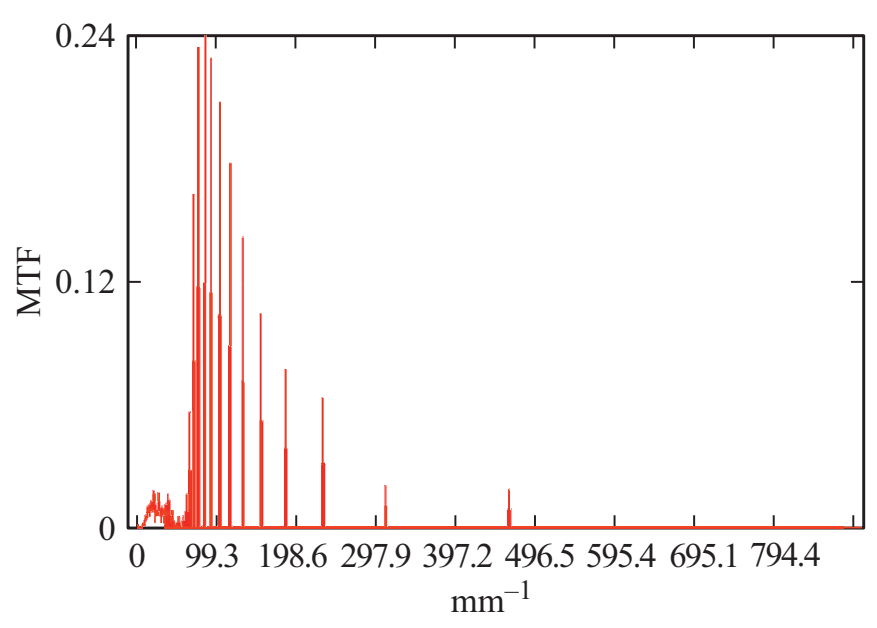

Рис. 8. Экспериментальная ЧКХ для гибридной системы (частоты даны в расчете на размер растра светочувствительной матрицы).

неточного экспериментального определения частоты в изображении ( $\pm 1-2$ пиксела).

\section{Выводы}

Гибридная система в виде рефракционнодифракционного дублета способна формировать ФРТ, сопоставимую по ширине дифракционному пределу в достаточно широком спектральном диапазоне, что подтверждается данными моделирования. Проведенный эксперимент доказывает существенное улучшение ЧКХ разработанной системы по сравнению с ЧКХ одиночной рефракционной линзы с тем же фокусным расстоянием, что позволяет подтвердить данные моделирования. Дальнейшее повышение качества изображения в такой оптической системе является чисто технической задачей по повышению точности формирования микрорельефа дифракционной линзы.

\section{Благодарности}

Авторы выражают благодарность ЦКПО „Нанофотоника и дифракционная оптика“ за предоставленное технологическое оборудование.

\section{Финансирование работы}

Исследование выполнено при финансовой поддержке гранта РНФ 20-69-47110 в части создания дифракционных линз, Министерства науки и высшего образования РФ в рамках выполнения работ по Государственному заданию ФНИЦ „Кристаллография и фотоника“ РАН (соглашение №007-ГЗ/Ч3363/26) в части исследования формирования изображений с помощью гибридного объектива.

\section{Конфликт интересов}

Авторы заявляют, что у них нет конфликта интересов.

\section{Список литературы}

[1] Meem M., Banerji S., Majumder A., Pies Ch., Oberbiermann T., Sensale-Rodriguez B., Menon R. // Appl. Phys. Lett. 2020. V. 117. P. 041101.

[2] Banerji S., Meem M., Majumder A., Vasquez F.G., Sensale-Rodriguez B., Menon R. // Optica. 2019. V. 6. N 6. P. 805.

[3] Park S., Lee G., Park B., Seo Y., Park C., Chun Y.T., Joo Ch., Rho J., Kim J.M., Hone J., Jun S.Ch. // Light. Sci. Appl. 2020. V. 5. N 9. P. 98.

[4] Lin H., Xu Zai-Quan, Cao G., Zhang Yu., Zhou J., Wang Z., Wan Zh., Liu Zh., Loh K.P., Qiu Cheng-Wei, Bao Q., Jia B. // Light. Sci. Appl. 2020. V. 11. N 9. P. 137.

[5] Banerji S., Cooke J., Sensale-Rodriguez B. // Sci. Rep. 2020. V. 10. P. 14608

[6] Greisukh G.I., Ezhov E.G., Antonov A.I., Danilov V.A., Usievich B.A. // Quant. Electron. 2020. V. 50. N 7. P. 623-628.

[7] Greisukh G.I., Danilov V.A., Stepanov S.A., Antonov A.I., Usievich B.A. // Opt. Spectrosc. 2018. V. 125. N 2. P. 232-237.

[8] Antonov A.I., Greisukh G.I., Ezhov E.G., Stepanov S.A. // Optoelectronics, Instrumentation and Data Processing. 2017. V. 53. N5. P. 421-430.

[9] Nikonorov A.V., Petrov M.V., Bibikov S.A., Yakimov P.Y., Kutikova V.V., Yuzifovich Y.V., Morozov A.A., Skidanov R.V., Kazanskiy N.L. // IEEE J. Selected Topics in Applied Earth Observations and Remote Sensing. 2018. V. 11. N 9. P. $3338-3348.8424456$.

[10] Mao S., Zhao J. // Appl. Opt. 2020. V. 59. N 20. P. 5888.

[11] Piao M., Zhang B., Dong K. // Opt. Express. 2020. V. 28. N 20. P. 29076.

[12] Choi H., Yoon Y.J., Kim B., Lee S.H., Kim W.C., Park N.Ch., Park Y.P., Kang S. // Jap. J. Appl. Phys. 2008. V. 47. N 8. P. 6678.

[13] Greisukh G.I., Ezhov E.G., Antonov A.I. // Computer Optics. 2020. V. 44. N 2. P. $177-184$.

[14] Koronkevich V.P., Lenkova G.A., Korolkov V.P., Poleschuk A.G., Iskakov I.A., Gutman A.S. // Computer Optics. 2008. V. 32. N 1. P. 50-58.

[15] Koronkevich V.P., Lenkova G.A., Korol'kov V.P., Iskakov I.A. // J. Optical Technology. 2007. V. 74. N 12. P. 818-822.

[16] Korolkov V.P., Nasyrov R.K., Sametov A.R., Suhih S.A. // Proc. of SPIE. 2011. V. 7957. P. 795710. 